\title{
Output-feedback adaptive optimal control of interconnected systems based on robust adaptive dynamic programming
}

\author{
Weinan Gao $^{\text {a }}$, Yu Jiang ${ }^{\text {a }}$, Zhong-Ping Jiang ${ }^{\text {a,b }}$, Tianyou Chai ${ }^{\text {b }}$

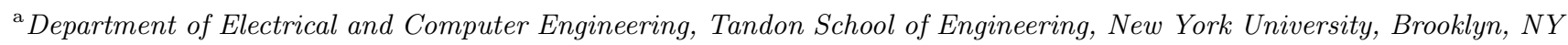 \\ 11201, USA \\ ${ }^{\mathrm{b}}$ State Key Lab of Synthetical Automation for Process Industries, Northeastern University, Shenyang, Liaoning 110819, China
}

\begin{abstract}
This paper studies the adaptive and optimal output-feedback problem for continuous-time uncertain systems with nonlinear dynamic uncertainties. Data-driven output-feedback control policies are developed by approximate/adaptive dynamic programming (ADP) based on both policy iteration and value iteration methods. The obtained adaptive and optimal outputfeedback controllers differ from the existing literature on the ADP in that they are derived from sampled-data systems theory and are guaranteed to be robust to dynamic uncertainties. A small-gain condition is given under which the overall system is globally asymptotically stable at the origin. An application to power systems is given to test the effectiveness of the proposed approaches.
\end{abstract}

Key words: Approximate/adaptive dynamic programming (ADP); Output-feedback control; Nonlinear dynamic uncertainty; Robust optimal control

\section{Introduction}

As an important subfield of modern control theory, optimal control aims to develop controllers that optimize certain performance (see Lewis et al., 2012a). Traditional optimal control methods need to solve the Hamilton-Jacobi-Bellman (HJB) equation (or algebraic Riccati equation (ARE) for linear systems) via the perfect knowledge of the system dynamics. Unfortunately, it is often difficult to obtain an accurate mathematical model for real-world, modern engineering and natural systems.

Approximate/adaptive dynamic programming (ADP) (see, e.g., Lewis and Liu, 2013; Si et al., 2004; Vamvoudakis, 2014; Zhang et al., 2013; Ni et al., 2013; Werbos, 1990, 1974) is a non-model-based approach which gives rise to online approximation of optimal solutions via some recursive numerical methods. The related

\footnotetext{
* This work has been supported in part by the U.S. National Science Foundation grant ECCS-1501044, and in part by the National Natural Science Foundation of China grant 61374042, and the Project 111 (No.B08015).

Email addresses: weinan.gao@nyu.edu (Weinan Gao), yu.jiang@nyu.edu (Yu Jiang), zjiang@nyu.edu (ZhongPing Jiang), tychai@mail.neu.edu.cn (Tianyou Chai).
}

research has been studied for discrete-time Markov decision processes (Powell, 2007; Bertsekas and Tsitsiklis, 1996; Sutton and Barto, 1998) and discrete-time feedback control systems (Lewis et al., 2012b; Lewis and Vrabie, 2009; Wang et al., 2009; Liu and Wei, 2014). For continuous-time systems, related work can be found in Vrabie et al. (2009); Jiang and Jiang (2012a, 2014); Bhasin et al. (2011); Luo et al. (2014); Yang et al. (2016). ADP and output regulation theory is firstly integrated by Gao and Jiang (2016) to solve the problem of asymptotic tracking with disturbance rejection.

Recently, extending these solutions to output-feedback problems has received attention from Lewis and Vamvoudakis (2011); Gao et al. (2014); Zhu et al. (2015); Gao et al. (2016) for linear systems and Liu et al. (2013); He and Jagannathan (2005) for nonlinear systems based on neural networks (Ge et al., 1998). A common feature of these papers is that no dynamic uncertainty (Jiang and Mareels, 1997) is addressed. However, there are numerous practical examples of continuous-time systems arising from engineering and biology for which dynamic uncertainty is unavoidable.

The contribution of this paper is threefold. First, different from existing output-feedback ADP for discretetime linear systems (Lewis and Vamvoudakis, 2011) 
or continuous-time static output-feedback ADP design which requires the accurate knowledge of the input matrix (Zhu et al., 2015), a dynamic output-feedback ADP approach is proposed for continuous-time linear systems without the exact knowledge of any system matrices. By employing the sampled-data system theory (Chen and Francis, 1995), the unmeasurable state can be reconstructed in terms of input/output data, whereby we can iteratively solve the ARE. Second, we, for the first time, develop an output-feedback value iteration (VI) ADP algorithm for continuous-time linear systems by using the sampled-data approach.

As the third contribution, we study the robust optimal redesign issue for a class of interconnected systems with dynamic uncertainties. The state and order of dynamic uncertainties are unknown. Because of the implementation of sampled-data output-feedback robust optimal controllers, the closed-loop system is a hybrid system that involves both continuous-time and discrete-time dynamics. Thus, robustness analysis cannot be conducted directly by our previous work on state-feedback robust ADP (Jiang and Jiang, 2014, 2013). Instead, we derive the global asymptotic stability of the closed-loop interconnected system based on a combined application of Lyapunov theory, sampled-data systems theory, and nonlinear small-gain method. To the best of the authors' knowledge, this paper represents the first step towards the ADP design of output-feedback adaptive optimal controllers for continuous-time nonlinear systems with both static and dynamic uncertainties.

The remainder of this paper is organized as follows. In Section 2, we formulate the control problem, and briefly review the linear-quadratic regulator (LQR) theory. In Section 3, we develop adaptive optimal output-feedback strategies by using both policy iteration (PI) and VI based ADP methods. Robustness and suboptimality of the closed-loop system are analyzed in Section 4. An application to a practical example on the power systems is presented in Section 5. Finally, conclusions are contained in Section 6.

Notations. Throughout this paper, $\mathbb{R}_{+}$denote the set of nonnegative real numbers. $|\cdot|$ represents the Euclidean norm for vectors and the induced norm for matrices. A continuous function $\alpha: \mathbb{R}_{+} \rightarrow \mathbb{R}_{+}$belongs to class $\mathcal{K}$ if it is increasing and $\alpha(0)=0$. It belongs to class $\mathcal{K}_{\infty}$ if, in addition, it is proper. A continuous function $\beta: \mathbb{R}_{+} \times \mathbb{R}_{+} \rightarrow \mathbb{R}_{+}$belongs to class $\mathcal{K} \mathcal{L}$ if for each fixed $t$, the function $\beta(\cdot, t)$ is of class $\mathcal{K}$ and, for each fixed $s$, the function $\beta(s, \cdot)$ is non-increasing and tends to 0 at infinity. $\otimes$ indicates the Kronecker product operator and $\operatorname{vec}(A)=\left[a_{1}^{T}, a_{2}^{T}, \cdots, a_{m}^{T}\right]^{T}$, where $a_{i} \in \mathbb{R}^{n}$ are the columns of $A \in \mathbb{R}^{n \times m}$. For a symmetric matrix $P \in \mathbb{R}^{m \times m}, \operatorname{vecs}(P)=$ $\left[p_{11}, 2 p_{12}, \cdots, 2 p_{1 m}, p_{22}, 2 p_{23}, \cdots, 2 p_{m-1, m}, p_{m m}\right]^{T} \in$ $\mathbb{R}^{\frac{1}{2} m(m+1)}$. For an arbitrary column vector $v \in \mathbb{R}^{n}$, $\operatorname{vecv}(v)=\left[v_{1}^{2}, v_{1} v_{2}, \cdots, v_{1} v_{n}, v_{2}^{2}, v_{2} v_{3}, \cdots, v_{n-1} v_{n}, v_{n}^{2}\right]^{T}$ $\in \mathbb{R}^{\frac{1}{2} n(n+1)} . \lambda_{M}(P)$ and $\lambda_{m}(P)$ denote the maximum and the minimum eigenvalue of the real symmetric matrix $P$. For any piecewise continuous function $u: \mathbb{R}_{+} \rightarrow \mathbb{R}^{m},\|u\|$ stands for $\sup _{t \geq 0}|u(t)|$.

\section{Problem formulation and preliminaries}

Consider a linear subsystem interacting with a nonlinear subsystem known as the dynamic uncertainty, characterized by the $\zeta$-system:

$$
\begin{aligned}
\dot{x} & =A x+B(u+\Delta(\zeta, y)), \\
\dot{\zeta} & =g(\zeta, y), \\
y & =C x
\end{aligned}
$$

where $x \in \mathbb{R}^{n}, \zeta \in \mathbb{R}^{p}$ are unmeasurable states with an unknown integer $p>0, u \in \mathbb{R}^{m}$ the input, $y \in$ $\mathbb{R}^{r}$ the output. $A \in \mathbb{R}^{n \times n}, B \in \mathbb{R}^{n \times m}$, and $C \in \mathbb{R}^{r \times n}$ are unknown system matrices with $(A, B)$ controllable, $(A, C)$ observable satisfying $|A| \leq A_{M},|B| \leq B_{M}$, and $|C| \leq C_{M} \cdot g: \mathbb{R}^{p} \times \mathbb{R}^{r} \rightarrow \mathbb{R}^{p}$ and $\Delta: \mathbb{R}^{p} \times \mathbb{R}^{r} \rightarrow \mathbb{R}^{m}$ are two unknown, locally Lipschitz functions with $g(0,0)=$ 0 and $\Delta(0,0)=0$.

Remark 2.1 The system (1)-(3) belongs to the class of interconnected systems studied by Saberi et al. (1990). If $\Delta(\zeta, y)=\Delta_{1}(y)$, the system (1) and (3) is a linear system with nonlinear output injection (see Krener and Isidori, 1983).

Assumption 2.1 The $\zeta$-system with y regarded as the input and $\Delta$ as the output has the strong unboundedness observability (SUO) property with zero offset (Jiang et al., 1994), i.e., there exist a function $\sigma_{1}$ of class $\mathcal{K} \mathcal{L}$ and a function $\gamma_{1}$ of class $\mathcal{K}$ such that for any measurable essentially bounded control $y(t)$ on $[0, T)$ with $0<T \leq+\infty$, the solution $\zeta(t)$ of (2) right maximally defined on $\left[0, T^{\prime}\right)\left(0<T^{\prime} \leq T\right)$ satisfies

$$
|\zeta(t)| \leq \sigma_{1}(|\zeta(0)|, t)+\gamma_{1}\left(\left\|\left[y_{[0, t]}^{T}, \Delta_{[0, t]}^{T}\right]^{T}\right\|\right), \forall t \in\left[0, T^{\prime}\right)
$$

where $y_{[0, t]}$ and $\Delta_{[0, t]}$ are truncated functions of $y$ and $\Delta$ over $[0, t]$, respectively.

Assumption 2.2 The $\zeta$-system is input-to-output stable (IOS) (Sontag, 2007), i.e., there exist a function $\sigma_{\Delta}$ of class $\mathcal{K} \mathcal{L}$ and a function $\gamma_{\Delta}$ of class $\mathcal{K}$ such that, for any initial state $\zeta(0)$, any measurable essentially bounded input $y$ and any $t \geq 0$,

$$
|\Delta(t)| \leq \sigma_{\Delta}(|\zeta(0)|, t)+\gamma_{\Delta}(\|y\|)
$$

Considering the reduced-order system (1) and (3) in the absence of the dynamic uncertainty

$$
\begin{aligned}
& \dot{x}=A x+B u, \\
& y=C x,
\end{aligned}
$$


define the cost as

$$
J_{c}(x(0))=\int_{0}^{\infty}\left(y^{T}(\tau) Q y(\tau)+u^{T}(\tau) R u(\tau)\right) d \tau
$$

where $Q=Q^{T} \geq 0$ and $R=R^{T}>0$ with $(A, \sqrt{Q} C)$ observable. Moreover, a minimal cost $J_{c}^{*}=x^{T}(0) P^{*} x(0)$ in (6) is obtained by using the following control policy

$$
u=-R^{-1} B^{T} P^{*} x:=-K^{*} x
$$

where $P^{*}=\left(P^{*}\right)^{T}>0$ is the unique solution to the algebraic Riccati equation (ARE):

$$
A^{T} P^{*}+P^{*} A+C^{T} Q C-P^{*} B R^{-1} B^{T} P^{*}=0 .
$$

A discretized model of (5) is obtained by taking periodic sampling

$$
\begin{aligned}
x_{k+1} & =A_{d} x_{k}+B_{d} u_{k}, \\
y_{k} & =C x_{k}
\end{aligned}
$$

where $A_{d}=e^{A h}, B_{d}=\left(\int_{0}^{h} e^{A \tau} d \tau\right) B$, and $h>0$ is the sampling period. Suppose the sampling frequency $\omega_{h}=2 \pi / h$ is non-pathological (Chen and Francis, 1995). Then, both $\left(A_{d}, C\right)$ and $\left(A_{d}, \sqrt{Q} C\right)$ are observable with $\left(A_{d}, B_{d}\right)$ controllable. The cost for $(9)$ is

$$
J_{d}(x(0))=\sum_{j=0}^{\infty}\left(y_{j}^{T} Q_{d} y_{j}+u_{j}^{T} R_{d} u_{j}\right)
$$

where $Q_{d}=Q h, R_{d}=R h$. Notice that (10) can be viewed as a first-order approximation of equation (7) in Melzer and Kuo (1971) which itself is the discretized version of cost (6).

The optimal control law minimizing (10) is

$$
u_{k}=-\left(R_{d}+B_{d}^{T} P_{d}^{*} B_{d}\right)^{-1} B_{d}^{T} P_{d}^{*} A_{d} x_{k}:=-K_{d}^{*} x_{k}
$$

where $P_{d}^{*}=P_{d}^{* T}>0$ is the unique solution to

$$
\begin{aligned}
& A_{d}^{T} P_{d}^{*} A_{d}-P_{d}^{*}+C^{T} Q_{d} C \\
& -A_{d}^{T} P_{d}^{*} B_{d}\left(R_{d}+B_{d}^{T} P_{d}^{*} B_{d}\right)^{-1} B_{d}^{T} P_{d}^{*} A_{d}=0 .
\end{aligned}
$$

The sensitivities of $P_{d}^{*}$ and $K_{d}^{*}$, with respect to sampling period $h$, are discussed in the following Lemma.

Lemma 2.1 Letting $P_{d}^{*}$ and $K_{d}^{*}$ satisfy equations (11)(12) at sampling period $h$, then

$$
\begin{aligned}
P_{d}^{*} & =P^{*}+\frac{1}{2} C^{T} Q C h+O_{P}\left(h^{2}\right), \\
K_{d}^{*} & =K^{*}+\frac{1}{2} K^{*}\left(A-B K^{*}\right) h+O_{K}\left(h^{2}\right)
\end{aligned}
$$

where, for $i=P, K, \limsup _{h \rightarrow 0}\left|O_{i}\left(h^{2}\right) / h^{2}\right|<\infty$.

Proof Inspired by Melzer and Kuo (1971), for $h>0$, let

$$
\begin{aligned}
X(h) & =\left(A_{d}^{T} P_{d}^{*}(h) A_{d}-P_{d}^{*}(h)+C^{T} Q_{d} C\right) / h, \\
Y(h) & =\left(A_{d}^{T} P_{d}^{*}(h) B_{d}\right) / h, \\
Z(h) & =\left(R_{d}+B_{d}^{T} P_{d}^{*}(h) B_{d}\right) / h .
\end{aligned}
$$

It is easy to obtain the limits of $X(h), Y(h)$ and $Z(h)$ as $h$ goes to zero:

$$
\begin{aligned}
& X(0)=P_{d}^{*}(0) A+A^{T} P_{d}^{*}(0)+C^{T} Q C, \\
& Y(0)=P_{d}^{*}(0) B, \quad Z(0)=R .
\end{aligned}
$$

Then, equations (11)-(12) imply

$$
\begin{aligned}
& K_{d}^{*}(h)=Z^{-1}(h) Y^{T}(h), K_{d}^{*}(0)=R^{-1} B^{T} P_{d}^{*}(0), \\
& X(h)=Y(h) K_{d}^{*}(h), P_{d}^{*}(0) A+A^{T} P_{d}^{*}(0) \\
& +C^{T} Q C=P_{d}^{*}(0) B R^{-1} B^{T} P_{d}^{*}(0)
\end{aligned}
$$

which indicates that $P_{d}^{*}(0)=P^{*}$ and $K_{d}^{*}(0)=K^{*}$. For the first-order sensitivities, $K_{d}^{*}(h)$ and $X(h)$ are differentiated at $h=0$,

$$
\begin{aligned}
\frac{\partial K_{d}^{*}}{\partial h} & =R^{-1} \frac{\partial Y^{T}}{\partial h}-R^{-1} \frac{\partial Z}{\partial h} R^{-1} B^{T} P_{d}^{*}(0), \\
\frac{\partial X}{\partial h} & =P_{d}^{*}(0) B \frac{\partial K_{d}^{*}}{\partial h}+\frac{\partial Y}{\partial h} R^{-1} B^{T} P_{d}^{*}(0) .
\end{aligned}
$$

By (14), the first-order sensitivities of $X(h), Y(h)$ and $Z(h)$ are

$$
\begin{aligned}
\frac{\partial X}{\partial h}= & A^{T} \frac{\partial P_{d}^{*}}{\partial h}+\frac{\partial P_{d}^{*}}{\partial h} A+\frac{A^{T}}{2}\left(A^{T} P_{d}^{*}(0)+P_{d}^{*}(0) A\right) \\
& +\left(A^{T} P_{d}^{*}(0)+P_{d}^{*}(0) A\right) \frac{A}{2} \\
\frac{\partial Y}{\partial h}= & \frac{\partial P_{d}^{*}}{\partial h} B+\frac{1}{2}\left(2 A^{T} P_{d}^{*}(0)+P_{d}^{*}(0) A\right) B \\
\frac{\partial Z}{\partial h}= & B^{T} P_{d}^{*}(0) B
\end{aligned}
$$

Substituting (17) into (16), and by (15), we have

$$
\begin{gathered}
\left(A-B R^{-1} B^{T} P_{d}^{*}(0)\right)^{T}\left(\frac{\partial P_{d}^{*}}{\partial h}-\frac{C^{T} Q C}{2}\right)+ \\
\left(\frac{\partial P_{d}^{*}}{\partial h}-\frac{C^{T} Q C}{2}\right)\left(A-B R^{-1} B^{T} P_{d}^{*}(0)\right)=0 \\
\frac{\partial K_{d}^{*}}{\partial h}=R^{-1} B^{T}\left(\frac{\partial P_{d}^{*}}{\partial h}-\frac{C^{T} Q C}{2}\right)+\frac{1}{2} K_{d}^{*}(0)\left(A-B K_{d}^{*}(0)\right) .
\end{gathered}
$$

Since $A-B R^{-1} B^{T} P_{d}^{*}(0)$ is asymptotically stable, we obtain $\frac{\partial P_{d}^{*}}{\partial h}=\frac{C^{T} Q C}{2}$ and $\frac{\partial K_{d}^{*}}{\partial h}=\frac{1}{2} K^{*}\left(A-B K^{*}\right)$. 
Remark 2.2 Lemma 2.1 implies that, for any $\epsilon_{1}>0$, there exists $\delta_{1}>0$ such that $\left|K^{*}-K_{d}^{*}\right|<\epsilon_{1}$, if the sampling period $h<\delta_{1}$.

The following technical theorem characterizes the relationship between the optimal cost for the original continuous-time system and the cost value for the discretized counterpart under the sampled-data controller (11).

Theorem 2.1 Letting $J_{c}^{\oplus}$ be the cost in (6) for the system (5) in closed-loop with controller (11), for small $h>0$ such that $\omega_{h}$ is non-pathological, the error between $J_{c}^{\oplus}$ and $J_{c}^{*}$ is

$$
J_{c}^{\oplus}-J_{c}^{*}=y^{T}(0) Q y(0) h+O_{J}\left(h^{2}\right)
$$

where $\limsup _{h \rightarrow 0}\left|O_{J}\left(h^{2}\right) / h^{2}\right|<\infty$.

Proof Write $J^{\oplus}$ as a summation (see, Melzer and Kuo, 1971):

$$
\begin{aligned}
J^{\oplus} & =\int_{0}^{\infty}\left(y^{T}(\tau) Q y(\tau)+u^{T}(\tau) R u(\tau)\right) d \tau \\
& =\sum_{j=0}^{\infty} x_{j}^{T} \hat{Q} x_{j}+2 x_{j}^{T} M u_{j}+u_{j}^{T} \hat{R} u_{j}
\end{aligned}
$$

where

$$
\begin{aligned}
\hat{Q} & =\int_{0}^{h}\left(e^{A s}\right)^{T} C^{T} Q C e^{A s} d s \\
M & =\int_{0}^{h}\left[\left(e^{A s}\right)^{T} C^{T} Q C\left(\int_{0}^{s} e^{A \lambda} B d \lambda\right)\right] d s \\
\hat{R} & =R_{d}+\int_{0}^{h}\left[\left(\int_{0}^{s} e^{A \lambda} B d \lambda\right)^{T} C^{T} Q C\left(\int_{0}^{s} e^{A \lambda} B d \lambda\right)\right] d s .
\end{aligned}
$$

Then, we have $J^{\oplus}=x^{T}(0) P^{\oplus}(h) x(0)$, where $P^{\oplus}(h)$ satisfies

$$
\begin{gathered}
\left(A_{d}-B_{d} K_{d}^{*}\right)^{T} P^{\oplus}\left(A_{d}-B_{d} K_{d}^{*}\right)-P^{\oplus}= \\
\hat{Q}-M K_{d}^{*}-\left(K_{d}^{*}\right)^{T} M^{T}+\left(K_{d}^{*}\right)^{T} \hat{R} K_{d}^{*} .
\end{gathered}
$$

Define $P_{\Delta}^{\oplus}(h)=P^{\oplus}(h)-P_{d}^{*}(h)$, and

$X_{2}(h)=\frac{\left(A_{d}-B_{d} K_{d}^{*}\right)^{T} P_{\Delta}^{\oplus}\left(A_{d}-B_{d} K_{d}^{*}\right)-P_{\Delta}^{\oplus},}{h}$,

$Y_{2}(h)=$

$\frac{\hat{Q}-C^{T} Q_{d} C-M K_{d}^{*}-\left(K_{d}^{*}\right)^{T} M^{T}+\left(K_{d}^{*}\right)^{T}\left(\hat{R}-R_{d}\right) K_{d}^{*}}{h}$.

Equations (12) and (19) imply $X_{2}(h)=Y_{2}(h)$. By $X_{2}(0)=Y_{2}(0)$ and the first-order sensitivities of $X_{2}$ and $Y_{2}$, we have

$$
\begin{aligned}
0= & \left(A-B K^{*}\right)^{T} P_{\Delta}^{\oplus}(0)+P_{\Delta}^{\oplus}(0)\left(A-B K^{*}\right), \\
0= & \left(A-B K^{*}\right)^{T}\left(\frac{\partial P_{\Delta}^{\oplus}}{\partial h}-\frac{C^{T} Q C}{2}\right)+ \\
& \left(\frac{\partial P_{\Delta}^{\oplus}}{\partial h}-\frac{C^{T} Q C}{2}\right)\left(A-B K^{*}\right) .
\end{aligned}
$$

$\left(A-B K^{*}\right)$ is a Hurwitz matrix, revealing that $P_{\Delta}^{\oplus}(0)=0$ and $\frac{\partial P_{\Delta}^{\oplus}}{\partial h}=\frac{C^{T} Q C}{2}$. By Lemma 2.1, we obtain the approximation of $P^{\oplus}-P^{*}$ :

$$
P^{\oplus}-P^{*}=P_{\Delta}^{\oplus}+\left(P_{d}^{*}-P^{*}\right) \simeq C^{T} Q C h
$$

The proof is thus completed.

Remark 2.3 Since (12) is nonlinear in $P_{d}$, solving $P_{d}^{*}$ directly from (12) is difficult. It can be approximated by the PI Algorithm 1 (Hewer, 1971) or the VI Algorithm 2 (Lancaster and Rodman, 1995, Chap. 17).

Remark 2.4 The sequences $\left\{P_{j}\right\}_{j=0}^{\infty}$ and $\left\{K_{j}\right\}_{j=1}^{\infty}$ computed from Algorithm 1 or 2 converge to $P_{d}^{*}$ and $K_{d}^{*}$, respectively (see Hewer, 1971; Lancaster and Rodman, 1995). Moreover, for $j=0,1,2, \cdots$, we know $A_{d}-B_{d} K_{j}$ is Schur, with $K_{j}$ computed by Algorithm 1.

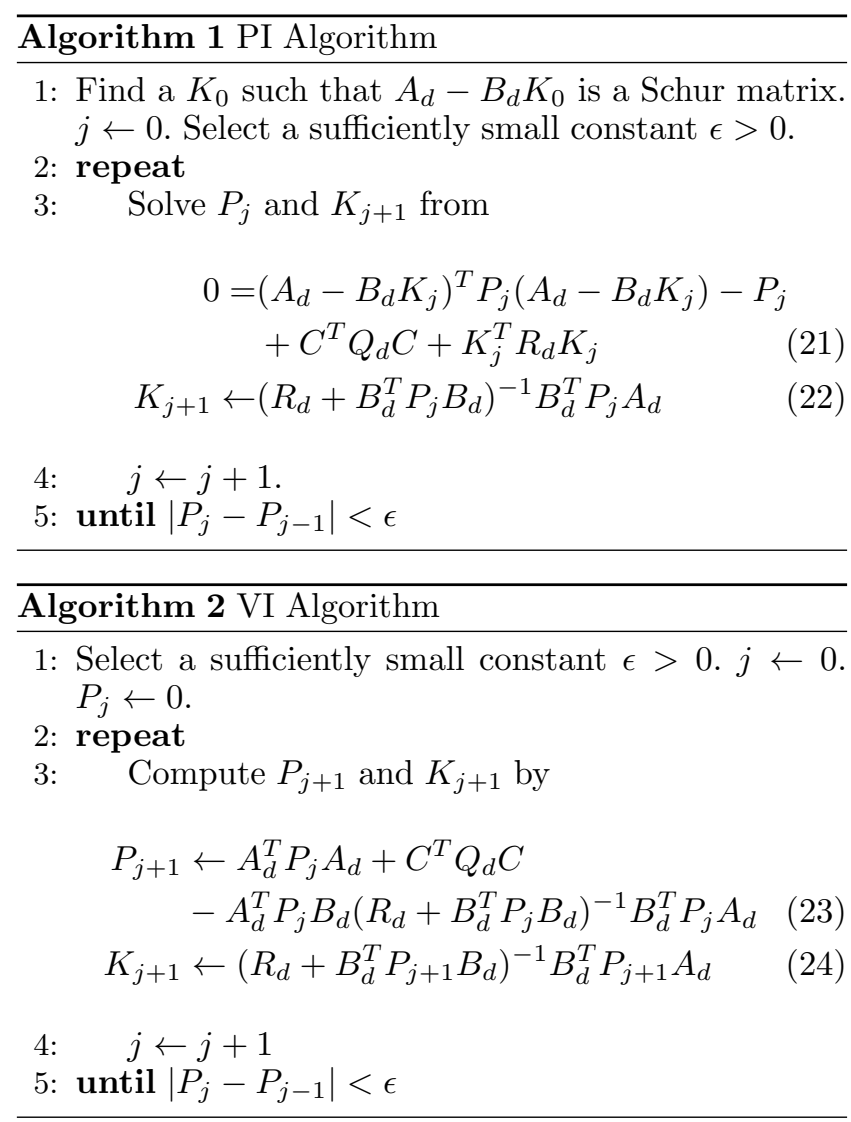




\section{$3 \quad$ ADP design for output-feedback control}

In this section, we present a state estimation method in terms of input/output data. Both PI-based and VIbased ADP learning strategies are proposed to find the optimal dynamic output-feedback controller with unknown system matrices.

\subsection{State estimation}

The discretized variant of system (1) and (3) can be rewritten as

$$
\begin{aligned}
x_{k+1} & =A_{d} x_{k}+B_{d} u_{k}+\xi_{k}, \\
y_{k} & =C x_{k}
\end{aligned}
$$

where $\xi_{k}=\int_{k h}^{(k+1) h} e^{A((k+1) h-\tau)} B \Delta(\zeta(\tau), y(\tau)) d \tau$. By Lewis and Vamvoudakis (2011); Aangenent et al. (2005), the state $x_{k}$ can be reconstructed as follows:

$$
x_{k}=M_{y} \bar{y}_{k}+M_{u} \bar{u}_{k}+M_{\xi} \bar{\xi}_{k}
$$

where

$$
\begin{aligned}
& M_{y}=A_{d}^{n}\left(U^{T} U\right)^{-1} U^{T} \in \mathbb{R}^{n \times r n} \\
& M_{u}=V-M_{y} S\left(I_{n} \otimes B_{d}\right) \in \mathbb{R}^{n \times m n} \\
& M_{\xi}=W-M_{y} S \in \mathbb{R}^{n \times n^{2}} \\
& \bar{u}_{k}=\left[u_{k-1}^{T}, u_{k-2}^{T}, \cdots, u_{k-n}^{T}\right]^{T} \in \mathbb{R}^{m n} \\
& \bar{y}_{k}=\left[y_{k-1}^{T}, y_{k-2}^{T}, \cdots, y_{k-n}^{T}\right]^{T} \in \mathbb{R}^{r n} \\
& \bar{\xi}_{k}=\left[\xi_{k-1}^{T}, \xi_{k-2}^{T}, \cdots, \xi_{k-n}^{T}\right]^{T} \in \mathbb{R}^{n^{2}} \\
& V=\left[B_{d}, A_{d} B_{d}, \cdots, A_{d}^{n-1} B_{d}\right] \in \mathbb{R}^{n \times m n} \\
& U=\left[\left(C A_{d}^{n-1}\right)^{T}, \cdots,\left(C A_{d}\right)^{T}, C^{T}\right]^{T} \in \mathbb{R}^{r n \times n} \\
& W=\left[I_{n}, A_{d}, \cdots, A_{d}^{n-1}\right] \in \mathbb{R}^{n \times n^{2}} \\
& S=\left[\begin{array}{ccccc}
0 & C & C A_{d} & \cdots & C A_{d}^{n-2} \\
0 & 0 & C & \cdots & C A_{d}^{n-3} \\
\vdots & \vdots & \ddots & \ddots & \vdots \\
0 & \cdots & & 0 & C \\
0 & 0 & 0 & 0 & 0
\end{array}\right] \in \mathbb{R}^{r n \times n^{2}}
\end{aligned}
$$

Suppose $\Delta(\zeta(t), y(t))$ is available in the learning phase. Let $u(t)=v(t)-\Delta(\zeta(t), y(t))$, where $v(t)$ is a piecewise constant signal. Defining the matrix $A_{j}:=A_{d}-B_{d} K_{j}$, by periodic sampling, we have

$$
\begin{aligned}
x_{k+1} & =A_{d} x_{k}+B_{d} v_{k}=A_{j} x_{k}+B_{d}\left(K_{j} x_{k}+v_{k}\right), \\
y_{k} & =C x_{k} .
\end{aligned}
$$

\subsection{PI-based output ADP design}

Letting $\bar{v}_{k}=\left[v_{k-1}^{T}, v_{k-2}^{T}, \cdots, v_{k-n}^{T}\right]^{T} \in \mathbb{R}^{m n}, z_{k}=$ $\left[\bar{v}_{k}^{T}, \bar{y}_{k}^{T}\right]^{T} \in \mathbb{R}^{q}, q=n(m+r), \Theta=\left[M_{u}, M_{y}\right], \bar{K}_{j}=K_{j} \Theta$ and $\bar{P}_{j}=\Theta^{T} P_{j} \Theta$, the state of (27) satisfies

$$
x_{k}=\Theta z_{k}
$$

From (21), (27) and (28), we have

$$
\begin{aligned}
& z_{k+1}^{T} \bar{P}_{j} z_{k+1}-z_{k}^{T} \bar{P}_{j} z_{k} \\
& =x_{k+1}^{T} P_{j} x_{k+1}-x_{k}^{T} P_{j} x_{k} \\
& =x_{k}^{T} A_{j}^{T} P_{j} A_{j} x_{k}+\left(K_{j} x_{k}+v_{k}\right)^{T} B_{d}^{T} P_{j} B_{d}\left(K_{j} x_{k}+v_{k}\right) \\
& +2\left(K_{j} x_{k}+v_{k}\right)^{T} B_{d}^{T} P_{j}\left(A_{d}-B_{d} K_{j}\right) x_{k}-x_{k}^{T} P_{j} x_{k} \\
& =\left(K_{j} x_{k}+v_{k}\right)^{T}\left[B_{d}^{T} P_{j} B_{d} B_{d}^{T} P_{j} A_{d}\right]\left[\begin{array}{c}
-K_{j} x_{k}+v_{k} \\
2 x_{k}
\end{array}\right] \\
& -\left(y_{k}^{T} Q_{d} y_{k}+x_{k}^{T} K_{j}^{T} R_{d} K_{j} x_{k}\right) \\
& =\left(\bar{K}_{j} z_{k}+v_{k}\right)^{T}\left[\begin{array}{ll}
\bar{H}_{j}^{11} & \bar{H}_{j}^{12}
\end{array}\right]\left[\begin{array}{c}
-\bar{K}_{j} z_{k}+v_{k} \\
2 z_{k}
\end{array}\right] \\
& -\left(y_{k}^{T} Q_{d} y_{k}+z_{k}^{T} \bar{K}_{j}^{T} R_{d} \bar{K}_{j} z_{k}\right)
\end{aligned}
$$

where $\bar{H}_{j}^{11}=B_{d}^{T} P_{j} B_{d}$ and $\bar{H}_{j}^{12}=B_{d}^{T} P_{j} A_{d} \Theta$.

For a sufficiently large positive integer $s$ and $j=$ $0,1,2, \cdots$, we define

$$
\begin{aligned}
\delta_{j, z z}= & {\left[\operatorname{vecv}\left(z_{k_{j, 0}+1}\right)-\operatorname{vecv}\left(z_{k_{j, 0}}\right), \operatorname{vecv}\left(z_{k_{j, 1}+1}\right)\right.} \\
& \left.-\operatorname{vecv}\left(z_{k_{j, 1}}\right), \cdots, \operatorname{vecv}\left(z_{k_{j, s}+1}\right)-\operatorname{vecv}\left(z_{k_{j, s}}\right)\right]^{T} \\
\Gamma_{j, z z}= & {\left[z_{k_{j, 0}} \otimes z_{k_{j, 0}}, z_{k_{j, 1}} \otimes z_{k_{j, 1}}, \cdots, z_{k_{j, s}} \otimes z_{k_{j, s}}\right]^{T} } \\
\Gamma_{j, z v}= & {\left[z_{k_{j, 0}} \otimes v_{k_{j, 0}}, z_{k_{j, 1}} \otimes v_{k_{j, 1}}, \cdots, z_{k_{j, s}} \otimes v_{k_{j, s}}\right]^{T} } \\
\Gamma_{j, \tilde{v}}= & {\left[\operatorname{vecv}\left(v_{k_{j, 0}}\right), \operatorname{vecv}\left(v_{k_{j, 1}}\right), \cdots, \operatorname{vecv}\left(v_{k_{j, s}}\right)\right]^{T} } \\
\Gamma_{j, \widetilde{k z}}= & {\left[\operatorname{vecv}\left(\bar{K}_{j} z_{k_{j, 0}}\right), \operatorname{vecv}\left(\bar{K}_{j} z_{k_{j, 1}}\right), \cdots, \operatorname{vecv}\left(\bar{K}_{j} z_{k_{j, s}}\right)\right]^{T} } \\
\Phi_{j}^{P}= & {\left[y_{k_{j, 0}}^{T} Q_{d} y_{k_{j, 0}}+z_{k_{j, 0}}^{T} \bar{K}_{j}^{T} R_{d} \bar{K}_{j} z_{k_{j, 0}}, \cdots, y_{k_{j, s}}^{T} Q_{d} y_{k_{j, s}}\right.} \\
& \left.+z_{k_{j, s}}^{T} \bar{K}_{j}^{T} R_{d} \bar{K}_{j} z_{k_{j, s}}\right]^{T}
\end{aligned}
$$

where

$$
n<k_{0,0}<k_{0,1}<\cdots<k_{0, s}<k_{0, s}+1=k_{1,0}<\cdots .
$$

For any stabilizing gain matrix $\bar{K}_{j},(29)$ indicates

$$
\Psi_{j}^{P}\left[\begin{array}{c}
\operatorname{vecs}\left(\bar{H}_{j}^{11}\right) \\
\operatorname{vec}\left(\bar{H}_{j}^{12}\right) \\
\operatorname{vecs}\left(\bar{P}_{j}\right)
\end{array}\right]=\Phi_{j}^{P}
$$

where $\Psi_{j}^{P}=\left[\Gamma_{j, \tilde{v}}-\Gamma_{j, \widetilde{k z}}, 2\left(\Gamma_{j, z v}+\Gamma_{j, z z}\left(I_{q} \otimes \bar{K}_{j}^{T}\right)\right),-\delta_{j, z z}\right] \in$ $\mathbb{R}^{(s+1) \times\left(\frac{1}{2} m(m+1)+m q+\frac{1}{2} q(q+1)\right)}$. 
Lemma 3.1 If there exists a positive integer $s^{*}$ such that for all $s>s^{*}$

$\operatorname{rank}\left(\left[\Gamma_{j, z z}, \Gamma_{j, z v}, \Gamma_{j, \tilde{v}}\right]\right)=\frac{q(q+1)}{2}+q m+\frac{m(m+1)}{2}$,

then, (30) has a unique solution.

Proof See Gao et al. (2014).

Under the condition of Lemma 3.1, equation (30) is uniquely solved by the least squares method. From (22), $\bar{K}_{j+1}$ is obtained by $\bar{H}_{j}^{11}$ and $\bar{H}_{j}^{12}$

$$
\bar{K}_{j+1}=\left(R+\bar{H}_{j}^{11}\right)^{-1} \bar{H}_{j}^{12} .
$$

Now, we are ready to present an online PI-based algorithm for the adaptive optimal output-feedback design.

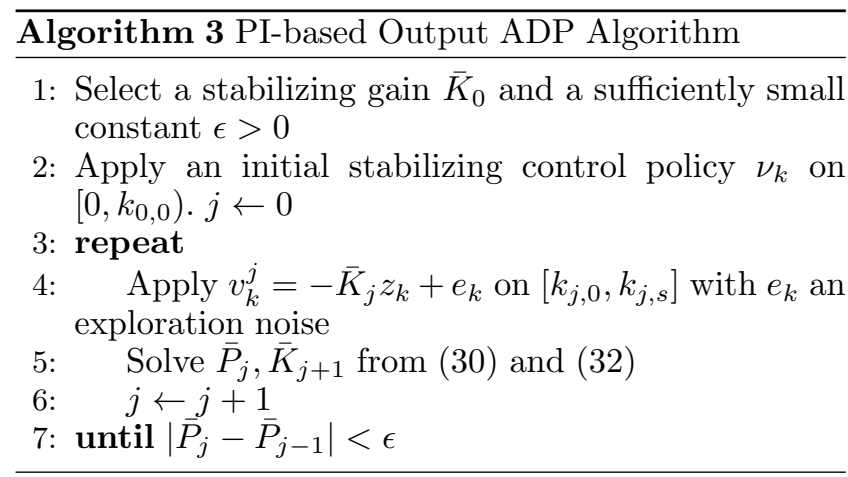

Theorem 3.1 If (31) is satisfied, sequences $\left\{\bar{P}_{j}\right\}_{j=0}^{\infty}$ and $\left\{\bar{K}_{j}\right\}_{j=1}^{\infty}$ obtained by Algorithm 3 converge to $\bar{P}_{d}^{*}$ and $\bar{K}_{d}^{*}$. where $\bar{P}_{d}^{*}=\Theta^{T} P_{d}^{*} \Theta$ and $\bar{K}_{d}^{*}=K_{d}^{*} \Theta$.

Proof Given a stabilizing $K_{j}$, if $P_{j}=P_{j}^{T}$ is the solution to (21), then $K_{j+1}$ is uniquely determined by (22). By (29), $\bar{P}_{j}$ and $\bar{K}_{j+1}$ satisfy (30) and (32). Letting $\bar{P}$ and $\bar{K}$ solve (30) and (32), (31) ensures that $\bar{P}_{j}=\bar{P}$ and $\bar{K}_{j+1}=\bar{K}$ are uniquely determined. By Hewer (1971), we have $\lim _{j \rightarrow \infty} \bar{K}_{j}=\bar{K}_{d}^{*}, \lim _{j \rightarrow \infty} \bar{P}_{j}=\bar{P}_{d}^{*}$.

\subsection{VI-based output ADP design}

Let two matrices, $H_{j}$ and $\bar{H}_{j}$, take the form of

$$
\begin{aligned}
H_{j} & =\left[\begin{array}{cc}
H_{j}^{11} & H_{j}^{12} \\
\left(H_{j}^{12}\right)^{T} & H_{j}^{22}
\end{array}\right] \equiv\left[\begin{array}{cc}
B_{d}^{T} P_{j} B_{d} & B_{d}^{T} P_{j} A_{d} \\
A_{d}^{T} P_{j} B_{d} & A_{d}^{T} P_{j} A_{d}
\end{array}\right], \\
\bar{H}_{j} & =\left[\begin{array}{cc}
\bar{H}_{j}^{11} & \bar{H}_{j}^{12} \\
\left(\bar{H}_{j}^{12}\right)^{T} & \bar{H}_{j}^{22}
\end{array}\right] \equiv\left[\begin{array}{cc}
B_{d}^{T} P_{j} B_{d} & B_{d}^{T} P_{j} A_{d} \Theta \\
\Theta^{T} A_{d}^{T} P_{j} B_{d} & \Theta^{T} A_{d}^{T} P_{j} A_{d} \Theta
\end{array}\right] .
\end{aligned}
$$

From (23) and (27), we obtain

$$
\begin{aligned}
& y_{k+1}^{T} Q_{d} y_{k+1} \\
= & -x_{k+1}^{T} \mathcal{F}\left(P_{j}\right) x_{k+1}+x_{k+1}^{T} P_{j+1} x_{k+1} \\
= & -x_{k+1}^{T}\left[H_{j}^{22}-\left(H_{j}^{12}\right)^{T}\left(R_{d}+H_{j}^{11}\right)^{-1} H_{j}^{12}\right] x_{k+1} \\
& +\left(\left[\begin{array}{l}
v_{k} \\
x_{k}
\end{array}\right] \otimes\left[\begin{array}{l}
v_{k} \\
x_{k}
\end{array}\right]\right)^{T} \operatorname{vec}\left(H_{j+1}\right) \\
= & -z_{k+1}^{T}\left[\bar{H}_{j}^{22}-\left(\bar{H}_{j}^{12}\right)^{T}\left(R+\bar{H}_{j}^{11}\right)^{-1} \bar{H}_{j}^{12}\right] z_{k+1} \\
& +\left[\operatorname{vecv}\left(\left[\begin{array}{l}
v_{k} \\
z_{k}
\end{array}\right]\right)\right]^{T} \operatorname{vecs}\left(\bar{H}_{j+1}\right) \\
= & -\phi_{k+1}^{j}+\left(\psi_{k}\right)^{T} \operatorname{vecs}\left(\bar{H}_{j+1}\right)
\end{aligned}
$$

where

$$
\begin{aligned}
\mathcal{F}\left(P_{j}\right) & =A_{d}^{T} P_{j} A_{d}-A_{d}^{T} P_{j} B_{d}\left(R_{d}+B_{d}^{T} P_{j} B_{d}\right)^{-1} B_{d}^{T} P_{j} A_{d}, \\
\phi_{k+1}^{j} & =z_{k+1}^{T}\left[\bar{H}_{j}^{22}-\left(\bar{H}_{j}^{12}\right)^{T}\left(R+\bar{H}_{j}^{11}\right)^{-1} \bar{H}_{j}^{12}\right] z_{k+1}, \\
\psi_{k} & =\operatorname{vecv}\left(\left[v_{k}^{T} z_{k}^{T}\right]^{T}\right) .
\end{aligned}
$$

Define

$$
\begin{gathered}
\Psi_{j}^{V}=\left[\psi_{k_{j, 0}}, \psi_{k_{j, 1}}, \cdots, \psi_{k_{j, s}}\right]^{T}, \\
\Phi_{j}^{V}=\left[y_{k_{j, 0}+1}^{T} Q_{d} y_{k_{j, 0}+1}+\phi_{k_{j, 0}+1}^{j}, \cdots,\right. \\
\left.y_{k_{j, s}+1}^{T} Q_{d} y_{k_{j, s}+1}+\phi_{k_{j, s}+1}^{j}\right]^{T} .
\end{gathered}
$$

(33) implies

$$
\Psi_{j}^{V} \operatorname{vecs}\left(\bar{H}_{j+1}\right)=\Phi_{j}^{V}
$$

whereof the uniqueness of the solution is also ensured by (31). The VI-based output ADP Algorithm 4 is developed as below. The convergence of Algorithm 4 is given in Theorem 3.2. The proof is similar to the proof of Theorem 3.1.

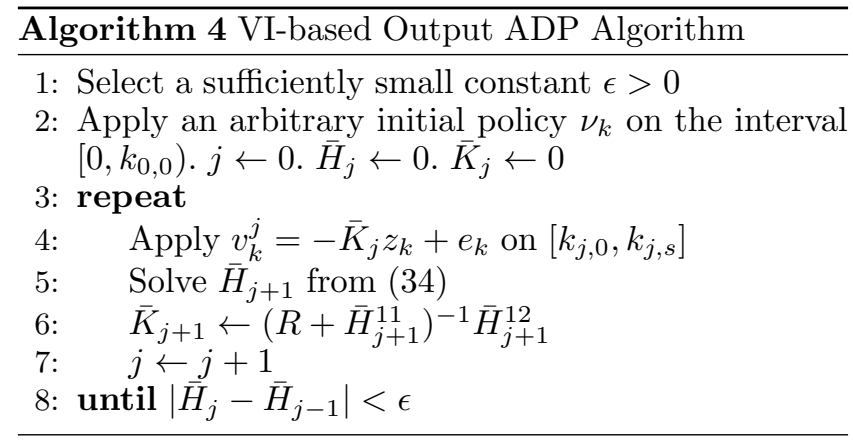

Theorem 3.2 If (31) holds, then $\lim _{j \rightarrow \infty} \bar{H}_{j}=\bar{H}_{d}^{*}$, 
$\lim _{j \rightarrow \infty} \bar{K}_{j}=\bar{K}_{d}^{*}$, where

$$
\bar{H}_{d}^{*}=\left[\begin{array}{cc}
B_{d}^{T} P_{d}^{*} B_{d} & B_{d}^{T} P_{d}^{*} A_{d} \Theta \\
\Theta^{T} A_{d}^{T} P_{d}^{*} B_{d} & \Theta^{T} A_{d}^{T} P_{d}^{*} A_{d} \Theta
\end{array}\right] .
$$

\section{Robustness and suboptimality}

In this section, we show the control policy

$$
u_{k}=-\bar{K}_{d}^{*}\left[\begin{array}{c}
\bar{u}_{k} \\
\bar{y}_{k}
\end{array}\right]
$$

obtained by Algorithms 3 and 4 robustly and globally asymptotically stabilizes the overall system (1)-(3) at the origin. The suboptimality of (35) is also analyzed.

\subsection{Robustness analysis}

Considering (1) in closed-loop with (35), by (26), it follows that

$$
\begin{aligned}
\dot{x}= & A x+B\left(u_{k}+\Delta\right) \\
= & \left(A-B K^{*}\right) x+B\left[\left(K^{*}-K_{d}^{*}\right) x+K_{d}^{*}\left(x-x_{k}\right)\right. \\
& \left.+K_{d}^{*} M_{\xi} \bar{\xi}_{k}+\Delta\right] .
\end{aligned}
$$

The following Lemma quantifies the dynamic uncertainty and the error between the state of the continuous system and that at the sampling instant.

Lemma 4.1 Let $t \in[k h,(k+1) h]$. For any $\epsilon_{2}>0$ and $\epsilon_{3}>1$, there exists a $\delta_{2}>0$ such that, if $h<\delta_{2}$,

$$
\begin{aligned}
\left|K_{d}^{*}\left(x(t)-x_{k}+M_{\zeta} \bar{\xi}_{k}\right)+\Delta\right| & \\
& \leq \epsilon_{2}|x(t)|+\epsilon_{3}\left\|\Delta_{[k h,(k+1) h]}\right\| .
\end{aligned}
$$

Proof Denote $x(t)$ by $x_{t}$ for simplicity. By (25) and (36), we have

$$
\begin{aligned}
\left|x_{t}-x_{k}\right| & \leq\left|\int_{0}^{h} e^{A \tau} d \tau\right|\left|A-B K_{d}^{*}\right|\left(\left|x_{t}-x_{k}\right|+\left|x_{t}\right|\right) \\
& +\left|\int_{0}^{h} e^{A \tau} d \tau\right||B|\left(\left|K_{d}^{*} M_{\zeta} \bar{\xi}_{k}\right|+\left\|\Delta_{[k h,(k+1) h]}\right\|\right) .
\end{aligned}
$$

Define $\phi_{1}(h)=h e^{A_{M} h}$. Let the sampling period $h$ satisfy

$$
\phi_{1}(h)\left(A_{M}+B_{M}\left|K_{d}^{*}\right|\right)<1,
$$

which implies $1-\left|\int_{0}^{h} e^{A \tau} d \tau\right|\left|A-B K_{d}^{*}\right|>0$. Then,

$$
\begin{aligned}
& \left|K_{d}^{*}\left(x_{t}-x_{k}+M_{\zeta} \bar{\xi}_{k}\right)+\Delta\right| \\
\leq & \frac{\left|\int_{0}^{h} e^{A \tau} d \tau\right|\left|A-B K_{d}^{*}\right|\left|K_{d}^{*}\right|}{1-\left|\int_{0}^{h} e^{A \tau} d \tau\right|\left|A-B K_{d}^{*}\right|}\left|x_{t}\right| \\
& +\left[\left(\frac{\left|\int_{0}^{h} e^{A \tau} d \tau\right||B|\left|K_{d}^{*}\right|^{2}\left|M_{\zeta}\right|}{1-\left|\int_{0}^{h} e^{A \tau} d \tau\right|\left|A-B K_{d}^{*}\right|}+\left|K_{d}^{*}\right|\left|M_{\zeta}\right|\right)\right. \\
& \left|\int_{0}^{h} e^{A \tau} d \tau\right||B| \sqrt{n}+1 \\
& \left.+\frac{\left|\int_{0}^{h} e^{A \tau} d \tau\right||B|\left|K_{d}^{*}\right|}{1-\left|\int_{0}^{h} e^{A \tau} d \tau \| A-B K_{d}^{*}\right|}\right]\left\|\Delta_{[k h,(k+1) h]}\right\| \\
:= & \epsilon_{2}^{\prime}\left|x_{t}\right|+\epsilon_{3}^{\prime}\left\|\Delta_{[k h,(k+1) h]}\right\|
\end{aligned}
$$

The estimate of the upper bound of $\left|M_{\xi}\right|$ is

$$
\begin{aligned}
\phi_{2}(h)=n & \frac{1-\left(\sqrt{n} e^{A_{M} h}\right)^{n}}{1-\sqrt{n} e^{A_{M} h}} \\
& +\sqrt{r n^{3} q} C_{M} \frac{1-\left(\sqrt{n} e^{A_{M} h}\right)^{n-1}}{1-\sqrt{n} e^{A_{M} h}} .
\end{aligned}
$$

By Lemma 2.1, for any $\epsilon_{1}^{\prime}>0$, there exist a $\delta_{1}^{\prime}>0$ such that, if $h<\delta_{1}^{\prime}$, we have $\left|K_{d}^{*}\right|<\left|K^{*}\right|+\left|K^{*}-K_{d}^{*}\right| \leq$ $\left|R^{-1}\right| B_{M}\left|P^{*}\right|+\epsilon_{1}^{\prime}:=K_{M}$. From (38) and (39), for any $\epsilon_{2}>0$ and $\epsilon_{3}>1$, if $h<\delta_{2}<\delta_{1}^{\prime}$, where $\delta_{2}$ meets

$$
\begin{aligned}
\phi_{1}\left(\delta_{2}\right) & <\min \left\{\frac{\epsilon_{2}}{\left(A_{M}+B_{M} K_{M}\right)\left(K_{M}+\epsilon_{2}\right)},\right. \\
& \left.\frac{1}{A_{M}+B_{M} K_{M}}, \frac{\epsilon_{3}-1}{\left(A_{M}\left(\epsilon_{3}-1\right)+B_{M} K_{M}\left(\epsilon_{3}+1\right)\right)}\right\},
\end{aligned}
$$
$\phi_{2}\left(\delta_{2}\right)<$

$$
\left(\frac{\epsilon_{3}-1}{2 \sqrt{n} B_{M} \phi_{1}(h)}-K_{M}\right) \frac{1-\phi_{1}(h)\left(A_{M}+B_{M} K_{M}\right)}{B_{M} K_{M}^{2} \phi_{1}(h)},
$$

then (37) holds .

Choose a Lyapunov function $V=x^{T} P_{1} x$, where $P_{1}=$ $P_{1}^{T}>0$ solves the following equation with $\omega>0$ :

$$
\begin{aligned}
& \left(A-B K^{*}\right)^{T} P_{1}+P_{1}\left(A-B K^{*}\right)+C^{T} Q C \\
& +K^{* T} R K^{*}+\omega I=0 .
\end{aligned}
$$

By (8) and (40), we get

$$
P_{\Delta}=\omega \int_{0}^{\infty} e^{\left(A-B K^{*}\right)^{T} t} e^{\left(A-B K^{*}\right) t} d t
$$

where $P_{\Delta}=P_{1}-P^{*}$. Then, we have $\left|P_{\Delta}\right|=\omega c^{* 2} /\left(2 \mu^{*}\right)$ by $\left|e^{\left(A-B K^{*}\right) t}\right| \leq c^{*} e^{-\mu^{*} t}$. The derivative of the Lyapunov function $\bar{V}$ at $k h \leq t \leq(k+1) h$ along the trajec- 
tory of the system (36) is

$$
\begin{aligned}
\dot{V}= & x^{T}\left[\left(A-B K^{*}\right)^{T} P_{1}+P_{1}\left(A-B K^{*}\right)\right] x \\
& +2 x^{T} P^{*} B\left[\left(K^{*}-K_{d}^{*}\right) x+K_{d}^{*}\left(x-x_{k}+M_{\zeta} \bar{\xi}_{k}\right)+\Delta\right] \\
& +2 x^{T} P_{\Delta} B\left[\left(K^{*}-K_{d}^{*}\right) x+K_{d}^{*}\left(x-x_{k}+M_{\zeta} \bar{\xi}_{k}\right)+\Delta\right] \\
\leq & -x^{T} C^{T} Q C x-x^{T} K^{* T} R K^{*} x-\omega|x|^{2}+2 x^{T}\left(K^{*}\right)^{T} \\
& R\left[\left(K^{*}-K_{d}^{*}\right) x+K_{d}^{*}\left(x-x_{k}+M_{\zeta} \bar{\xi}_{k}\right)+\Delta\right] \\
& +|x|^{2}\left[\left(\frac{\omega c^{* 2} B_{M}}{\mu^{*}}\right)\left(\epsilon_{1}+\epsilon_{2}\right)+\frac{\omega^{2} c^{* 4} B_{M}^{2} \alpha}{4 \mu^{* 2}}\right] \\
& +\left\|\Delta_{[k h,(k+1) h]}\right\|^{2} \frac{\epsilon_{3}^{2}}{\alpha} \\
\leq & -d_{1}|x|^{2}+d_{2}\left\|\Delta_{[k h,(k+1) h]}\right\|^{2}
\end{aligned}
$$

where $\alpha>0$ and

$$
\begin{aligned}
d_{1}= & \omega-\frac{\omega c^{* 2} B_{M}}{\mu^{*}}\left(\epsilon_{1}+\epsilon_{2}\right)-3|R|\left(\epsilon_{1}^{2}+\epsilon_{2}^{2}\right) \\
& -\frac{\omega^{2} c^{* 4} B_{M}^{2} \alpha}{4 \mu^{* 2}}, \\
d_{2}= & 3|R| \epsilon_{3}^{2}+\frac{\epsilon_{3}^{2}}{\alpha} .
\end{aligned}
$$

We select $\omega, \alpha, \epsilon_{1}, \epsilon_{2}$ such that $d_{1}>0$. Then, for all $t \geq 0$, (41) implies directly that

$V(t) \leq \exp \left(-\frac{d_{1}}{\lambda_{m}\left(P^{*}\right)} t\right) V(0)+\frac{d_{2} \lambda_{m}\left(P^{*}\right)}{d_{1}}\|\Delta\|^{2}$.

An immediate consequence of the previous inequality is

$$
\begin{aligned}
|x(t)| \leq & \exp \left(-\frac{d_{1}}{2 \lambda_{m}\left(P^{*}\right)} t\right) \sqrt{\frac{\lambda_{M}\left(P^{*}\right)}{\lambda_{m}\left(P^{*}\right)}}|x(0)| \\
& +\sqrt{\frac{d_{2}}{d_{1}}}\|\Delta\|, \quad \forall t \geq 0
\end{aligned}
$$

which implies that the $x$-system, as written in (1) and (3), with $\Delta$ as the input is input-to-state stable (ISS) (Sontag, 1989). By (3), we have

$$
|y(t)| \leq \sigma_{y}(|x(0)|, t)+\gamma_{y}(\|\Delta\|)
$$

where $\sigma_{y}(|x(0)|, t)=C_{M} \exp \left(-\frac{d_{1}}{2 \lambda_{m}\left(P^{*}\right)} t\right) \sqrt{\frac{\lambda_{M}\left(P^{*}\right)}{\lambda_{m}\left(P^{*}\right)}}|x(0)|$ is a function of $\mathcal{K} \mathcal{L}$ and $\gamma_{y}(s)=C_{M} \sqrt{\frac{d_{2}}{d_{1}}} s$ is a function of $\mathcal{K}$. By (42) and (43), we have

$$
\gamma_{y}(s)=\left[C_{M} \frac{c^{* 2} B_{M} \epsilon_{3}}{\mu^{*}-c^{* 2} B_{M}\left(\epsilon_{1}+\epsilon_{2}\right)}+\epsilon_{4}\right] s
$$

where $\epsilon_{4}>0$.

Now, we are ready to give a sufficient condition under which the interconnected system (1)-(3) is globally asymptotically stable at the origin.

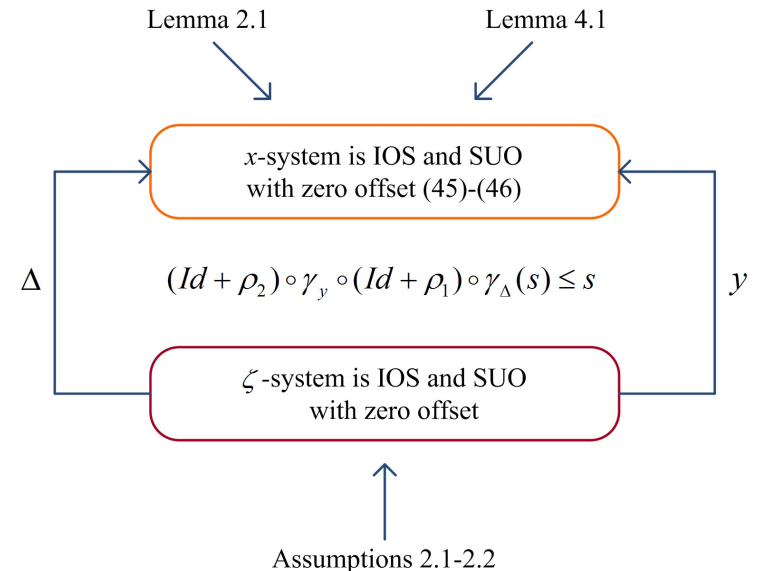

Fig. 1. The structure of the proof of Theorem 4.1

Theorem 4.1 Under the condition of Assumptions 2.1 and 2.2, if $h<\min \left\{\delta_{1}, \delta_{2}\right\}$, and $\gamma_{\Delta}(s)$ satisfies

$\gamma_{\Delta}(s) \leq\left(I d+\rho_{1}\right)^{-1} \circ \gamma_{y}^{-1} \circ\left(I d+\rho_{2}\right)^{-1}(s), \forall s \geq 0$

for some $\rho_{1}, \rho_{2}$ of class $\mathcal{K}_{\infty}$, the control policy (35) globally asymptotically stabilizes (1)-(3).

Proof Assumptions 2.1 and 2.2 indicate that the $\zeta$ system has SUO property with zero offset and is IOS with the gain function $\gamma_{\Delta}(s)$. (45) and (46) guarantee that the $x$-system has SUO property with zero offset and is IOS with the gain function $\gamma_{y}(s)$ (see Proposition 3.1, Jiang et al., 1994). By the nonlinear small-gain theory (Theorem 2.1, Jiang et al., 1994), the system (1)-(3) in closed-loop with (35) is globally asymptotically stable (GAS) at the origin under the following small-gain condition

$$
\left(I d+\rho_{2}\right) \circ \gamma_{y} \circ\left(I d+\rho_{1}\right) \circ \gamma_{\Delta}(s) \leq s, \forall s \geq 0 .
$$

The proof is thus completed. Fig. 1 is depicted to outline the structure of the proof.

\subsection{Suboptimality analysis}

If (48) holds, there exists a function $\sigma$ of class $\mathcal{K} \mathcal{L}$ such that $|\Delta(t)| \leq \sigma\left(\left|\left[x(0)^{T}, \zeta(0)^{T}\right]^{T}\right|, t\right)$. Letting $J_{c}^{\odot}$ be the cost in (6) for the closed-loop system (36) in the presence 
of dynamic uncertainty, we have

$$
\begin{aligned}
J_{c}^{\odot}= & \int_{0}^{\infty} y^{T} Q y+x^{T}\left(K^{*}\right)^{T} R K^{*} x d \tau \\
& +\left(u_{k}+K^{*} x\right)^{T} R\left(u_{k}+K^{*} x\right) d \tau \\
\leq & \int_{0}^{\infty}|x|^{2}\left|C^{T} Q C+\left(K^{*}\right)^{T} R K^{*}\right| d \tau \\
& +|R| \int_{0}^{\infty}\left[\left(\epsilon_{1}+\epsilon_{2}\right)|x|+\left(\epsilon_{3}-1\right)\left\|\Delta_{[\tau, \tau+h]}\right\|\right]^{2} d \tau \\
\leq & \int_{0}^{\infty}|x|^{2}\left[\left|C^{T} Q C+\left(K^{*}\right)^{T} R K^{*}\right|+2\left(\epsilon_{1}+\epsilon_{2}\right)^{2}|R|\right] d \tau \\
& +2\left(\epsilon_{3}-1\right)^{2}|R| h \sum_{i=0}^{\infty} \sigma^{2}(|[x(0)]|, i h) \cdot(49)
\end{aligned}
$$

(41) implies that

$$
\begin{aligned}
& x(k h+h)^{T} P_{1} x(k h+h)-x(k h)^{T} P_{1} x(k h) \\
& \quad \leq-d_{1} \int_{k h}^{(k+1) h}|x|^{2} d \tau+d_{2} h\left\|\Delta_{[k h,(k+1) h]}\right\|^{2}
\end{aligned}
$$

An immediate consequence is

$$
\begin{aligned}
& \int_{0}^{\infty}|x|^{2} d \tau \leq \\
& \frac{1}{d_{1}}\left\{d_{2} h \sum_{i=0}^{\infty} \sigma^{2}\left(\left|\left[\begin{array}{c}
x(0) \\
\zeta(0)
\end{array}\right]\right|, i h\right)+J_{c}^{*} \frac{\lambda_{M}\left(P_{1}\right)}{\lambda_{m}\left(P^{*}\right)}\right\}
\end{aligned}
$$

Combining (49) and (50), we obtain the following theorem that characterizes the suboptimality of the closedloop system composed of (1) and (35).

Theorem 4.2 Under the conditions of Assumptions 2.1 and 2.2, if (48) holds, then (35) is suboptimal for system (1)-(3) with the cost $J_{c}^{\odot}$ in (6) satisfying

$$
J_{c}^{\odot} \leq \eta J_{c}^{*}+D
$$

where

$$
\begin{aligned}
\eta= & \frac{\left[\left|C^{T} Q C+\left(K^{*}\right)^{T} R K^{*}\right|+2\left(\epsilon_{1}+\epsilon_{2}\right)^{2}|R|\right] \lambda_{M}\left(P_{1}\right)}{d_{1} \lambda_{m}\left(P^{*}\right)}, \\
D= & \left\{\frac{\left[\left|C^{T} Q C+\left(K^{*}\right)^{T} R K^{*}\right|+2\left(\epsilon_{1}+\epsilon_{2}\right)^{2}|R|\right] d_{2}}{d_{1}}\right. \\
& \left.+2\left(\epsilon_{3}-1\right)^{2}|R|\right\} \times h \sum_{i=0}^{\infty} \sigma^{2}\left(\left|\left[\begin{array}{c}
x(0) \\
\zeta(0)
\end{array}\right]\right|, i h\right) .
\end{aligned}
$$

\section{Application to Power Systems}

The stability analysis and controller design of power systems have attracted considerable attention recently (see,

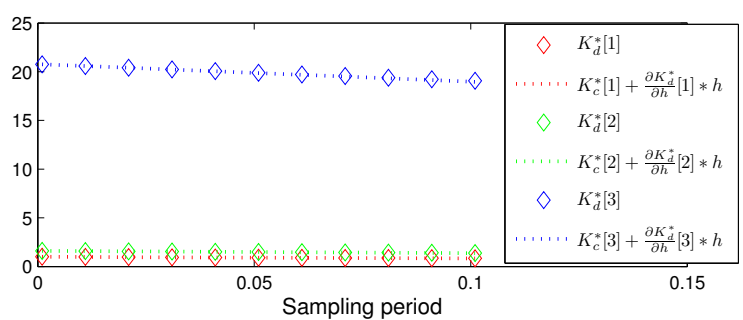

Fig. 2. Comparisons of $K_{d}^{*}$ and $K_{c}^{*}+\frac{\partial K_{d}^{*}}{\partial h} h$ at different $h$

e.g., Vrabie et al., 2009; Wang et al., 1993; Wang and Hill, 1996). In this section, we consider an interconnection of two synchronous generators wherein the generator 2 is regarded as the dynamic uncertainty of generator 1. The mathematical model of generators $i=1,2$ is

$$
\begin{aligned}
\Delta \dot{\delta}_{i}(t) & =-\Delta \omega_{i}(t), \\
\Delta \dot{\omega}_{i}(t) & =-\frac{D_{i}}{2 H_{i}} \Delta \omega_{i}(t)+\frac{\omega_{0}}{2 H_{i}} \Delta P_{m i}(t), \\
\Delta \dot{P}_{m i}(t) & =\frac{1}{T_{i}}\left[-\Delta P_{m i}(t)+u_{i}(t)-d(t)\right]
\end{aligned}
$$

where

$$
\begin{aligned}
d(t) & =E_{q 1}^{\prime} E_{q 2}^{\prime}\left[B_{12} \cos \left(\delta_{12}\right)-G_{12} \sin \left(\delta_{12}\right)\right]\left(\Delta \omega_{1}-\Delta \omega_{2}\right), \\
\delta_{12}(t) & =\left(\Delta \delta_{1}(t)+\delta_{10}\right)-\left(\Delta \delta_{2}(t)+\delta_{20}\right) .
\end{aligned}
$$

The meaning and value of parameters are referred to Jiang and Jiang (2012b). The system (52) takes the form of (1)-(3) with $x=\left[\Delta \delta_{1}, \Delta \omega_{1}, \Delta P_{m 1}\right]^{T}$, $y=\left[\Delta \delta_{1}, \Delta \omega_{1}\right]^{T}, \zeta=\left[\Delta \delta_{2}, \Delta \omega_{2}, \Delta P_{m 2}\right]^{T}, d=\Delta(\zeta, y)$. In order to validate Lemma 2.1 , Fig. 2 is depicted to show that, for small $h \in(0,0.1), K_{d}^{*}(h)$ is close to its first-order approximation computed by (13). In this example, $u_{2}(t)$ is already designed to satisfy Assumptions 2.1 and 2.2. $Q$ and $R$ are identity matrices, and $h=1 \mathrm{~ms}$. Both PI-based and VI-based output ADP learning algorithms are validated. Figs. 3 shows the convergence of $\bar{P}_{j}$ and $\bar{H}_{j}$ using Algorithms $3-4$. The plots of the angles and frequencies of generators 1 and 2 are depicted in Fig. 4.

\section{Conclusions}

This paper studies the adaptive optimal control problem for a class of continuous-time uncertain interconnected systems using output-feedback and adaptive dynamic programming. Both the PI-based and VI-based approaches are given for the iterative, online adaptive optimal output-feedback controller design. Robustness to dynamic uncertainties is examined by means of sampled-data systems theory, input-to-state stability and small-gain techniques. A practical application to power systems is given to validate the effectiveness of the proposed design. Our future work includes extensions to a broader class of nonlinear systems (Bian et al., 

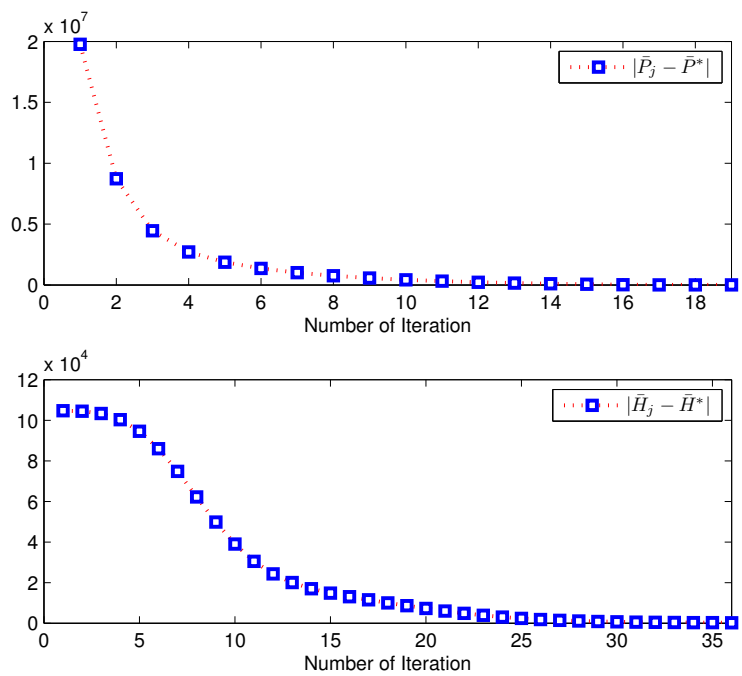

Fig. 3. Comparisons of $\bar{P}_{j}$ and $\bar{H}_{j}$ with their optimal values by Algorithms 3-4
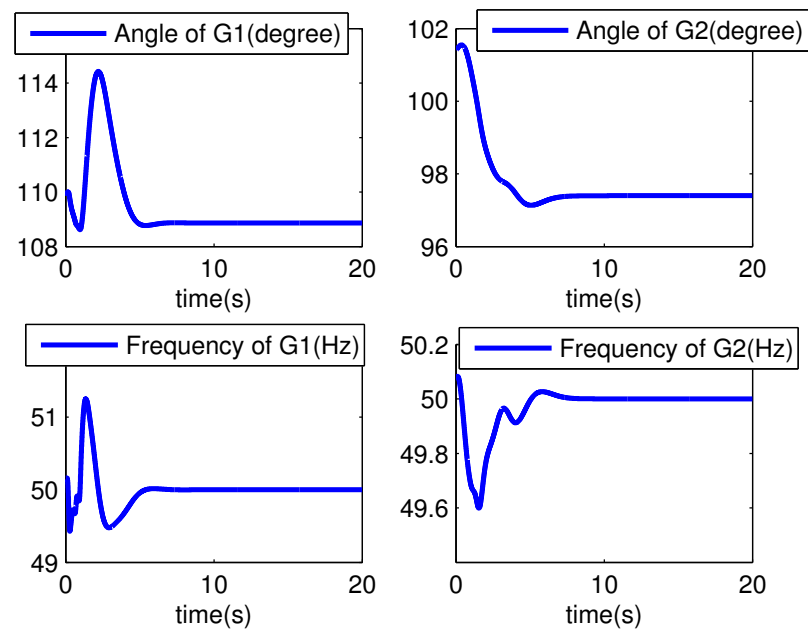

Fig. 4. Power angles and frequencies of generators

2014; Jiang and Jiang, 2015, 2014) and applications to intelligent transportation systems (Liu et al., 2016; Gao et al., 2015).

Acknowledgment. The authors thank Prof. A. Astolfi and Tao Bian for fruitful discussions.

\section{References}

Aangenent, W., Kostic, D., de Jager, B., van de Molengraft, R., Steinbuch, M., 2005. Data-based optimal control. In: Proceedings of the American Control Conference. Vol. 2. Portland, OR, pp. 1460-1465.

Bertsekas, D. P., Tsitsiklis, J. N., 1996. Neuro-Dynamic Programming. Athena Scientific, Belmont, MA.
Bhasin, S., Sharma, N., Parte, P., Dixon, W. E., 2011. Asymptotic tracking by a reinforcement learningbased adaptive critic controller. Journal of Control Theroy and Applications 9 (3), 400-409.

Bian, T., Jiang, Y., Jiang, Z. P., 2014. Adaptive dynamic programming and optimal control of nonlinear nonaffine systems. Automatica 50 (10), 2624 - 2632.

Chen, T., Francis, B. A., 1995. Optimal Sampled-data Control Systems. Springer.

Gao, W., Huang, M., Jiang, Z. P., Chai, T., 2016. Sampled-data-based adaptive optimal outputfeedback control of a 2-DOF helicopter. IET Control Theory and Applications, DOI: 10.1049/ietcta.2015.0977.

Gao, W., Jiang, Y., Jiang, Z. P., Chai, T., 2014. Adaptive and optimal output feedback control of linear systems: An adaptive dynamic programming approach. In: Proceedings of the 11th World Congress on Intelligent Control and Automation. Shenyang, China, pp. 2085-2090.

Gao, W., Jiang, Z. P., 2016. Adaptive dynamic programming and adaptive optimal output regulation of linear systems. IEEE Transactions on Automatic Control, DOI:10.1109/TAC.2016.2548662.

Gao, W., Jiang, Z. P., Ozbay, K., 2015. Adaptive optimal control of connected vehicles. In: Proceedings of the 10th International Workshop on Robot Motion and Control. Poznan, Poland, pp. 288-293.

Ge, S. S., Lee, T. H., Harris, C. J., 1998. Adaptive Neural Network Control of Robotic Manipulators. World Scientific Pub. Co. Inc.

He, P., Jagannathan, S., 2005. Reinforcement learningbased output feedback control of nonlinear systems with input constraints. IEEE Transactions on Systems, Man, and Cybernetics, Part B: Cybernetics 35 (1), 150-154.

Hewer, G., 1971. An iterative technique for the computation of the steady state gains for the discrete optimal regulator. IEEE Transactions on Automatic Control 16 (4), 382-384.

Jiang, Y., Jiang, Z. P., 2012a. Computational adaptive optimal control for continuous-time linear systems with completely unknown dynamics. Automatica 48 (10), 2699-2704.

Jiang, Y., Jiang, Z. P., 2012b. Robust adaptive dynamic programming for large-scale systems with an application to multimachine power systems. IEEE Transactions on Circuits and Systems II: Express Briefs 59 (10), 693-697.

Jiang, Y., Jiang, Z. P., 2014. Robust adaptive dynamic programming and feedback stabilization of nonlinear systems. IEEE Transactions on Neural Networks and Learning Systems 25 (5), 882-893.

Jiang, Y., Jiang, Z. P., 2015. Global adaptive dynamic programming for continuous-time nonlinear systems. IEEE Transactions on Automatic Control 60 (11), 2917-2929.

Jiang, Z. P., Jiang, Y., 2013. Robust adaptive dynamic programming for linear and nonlinear systems: An 
overview. European Journal of Control 19 (5), 417425.

Jiang, Z. P., Mareels, I., 1997. A small-gain control method for nonlinear cascaded systems with dynamic uncertainties. IEEE Transactions on Automatic Control 42 (3), 292-308.

Jiang, Z. P., Teel, A. R., Praly, L., 1994. Small-gain theorem for ISS systems and applications. Mathematics of Control, Signals and Systems 7 (2), 95-120.

Krener, A. J., Isidori, A., 1983. Linearization by output injection and nonlinear observers. Systems and Control Letters 3 (1), 47-52.

Lancaster, P., Rodman, L., 1995. Algebraic Riccati Equations. Oxford University Press Inc., New York, NY.

Lewis, F. L., Liu, D. (Eds.), 2013. Reinforcement Learning and Approximate Dynamic Programming for Feedback Control. Wiley, Hoboken, NJ.

Lewis, F. L., Vamvoudakis, K. G., 2011. Reinforcement learning for partially observable dynamic processes: Adaptive dynamic programming using measured output data. IEEE Transactions on Systems, Man, and Cybernetics, Part B: Cybernetics 41 (1), 14-25.

Lewis, F. L., Vrabie, D., 2009. Reinforcement learning and adaptive dynamic programming for feedback control. IEEE Circuits and Systems Magazine 9 (3), 3250.

Lewis, F. L., Vrabie, D., Syrmos, V. L., 2012a. Optimal Control. Wiley, Hoboken, NJ.

Lewis, F. L., Vrabie, D., Vamvoudakis, K. G., 2012 b. Reinforcement learning and feedback control: Using natural decision methods to design optimal adaptive controllers. IEEE Control Systems Magazine 32 (6), 76-105.

Liu, D., Huang, Y., Wang, D., Wei, Q., 2013. Neuralnetwork-observer-based optimal control for unknown nonlinear systems using adaptive dynamic programming. International Journal of Control 86 (9), 15541566.

Liu, D., Wei, Q., 2014. Policy iteration adaptive dynamic programming algorithm for discrete-time nonlinear systems. IEEE Transactions on Neural Networks and Learning Systems 25 (3), 621-634.

Liu, T., Lu, X., Jiang, Z. P., 2016. A junction-by-junction feedback-based strategy with convergence analysis for dynamic traffic assignment. Science China Information Sciences 59 (1), 1-17.

Luo, B., Wu, H. N., Huang, T., Liu, D., 2014. Databased approximate policy iteration for affine nonlinear continuous-time optimal control design. Automatica 50 (12), $3281-3290$.

Melzer, S. M., Kuo, B. C., 1971. Sampling period sensitivity of the optimal sampled data linear regulator. Automatica 7 (3), $367-370$.

Ni, Z., He, H., Wen, J., 2013. Adaptive learning in tracking control based on the dual critic network design. IEEE Transactions on Neural Networks and Learning Systems 24 (6), 913-928.

Powell, W. B., 2007. Approximate Dynamic Program- ming: Solving the curse of dimensionality. John Wiley \& Sons, New York, NY.

Saberi, A., Kokotovic, P., Summers, S., 1990. Global stabilization of partially linear composite systems. SIAM Journal of Control and Optimization 2 (6), 1491-1503.

Si, J., Barto, A. G., Powell, W. B., Wunsch, D. C., 2004. Handbook of Learning and Approximate Dynamic Programming. John Wiley \& Sons, New York, NY.

Sontag, E. D., 1989. Smooth stabilization implies coprime factorization. IEEE Transactions on Automatic Control 34 (4), 435-443.

Sontag, E. D., 2007. Input to state stability: Basic concepts and results. In: Nistri, P., Stefani, G. (Eds.), Nonlinear and Optimal Control Theory. SpringerVerlag, Berlin, pp. 163-220.

Sutton, R. S., Barto, A. G., 1998. Introduction to Reinforcement Learning. MIT Press, Cambridge, MA.

Vamvoudakis, K. G., 2014. Event-triggered optimal adaptive control algorithm for continuous-time nonlinear systems. IEEE/CAA Journal of Automatica Sinica (3), 282-293.

Vrabie, D., Pastravanu, O., Abu-Khalaf, M., Lewis, F., 2009. Adaptive optimal control for continuous-time linear systems based on policy iteration. Automatica 45 (2), $477-484$.

Wang, F. Y., Zhang, H., Liu, D., 2009. Adaptive dynamic programming: An introduction. IEEE Computational Intelligence Magazine 4 (2), 39-47.

Wang, Y., Hill, D. J., 1996. Robust nonlinear coordinated control of power systems. Automatica 32 (4), $611-618$.

Wang, Y., Zhou, R., Wen, C., 1993. Robust loadfrequency controller design for power systems. IEE Proceedings on Generation, Transmission and Distribution 140 (1), 11-16.

Werbos, P. J., 1974. Beyond regression: New tools for prediction and analysis in the behavioral sciences. Ph.D. thesis, Harvard University.

Werbos, P. J., 1990. A menu of designs for reinforcement learning over time. In: Miller, W., Sutton, R., Werbos, P. (Eds.), Neural Networks for Control. MIT Press, pp. 67-95.

Yang, X., Liu, D., Ma, H., Xu, Y., 2016. Online approximate solution of HJI equation for unknown constrained-input nonlinear continuous-time systems. Science China Information Sciences 328, 435 - 454.

Zhang, H., Liu, D., Luo, Y., Wang, D., 2013. Adaptive Dynamic Programming for Control: Algorithms and Stability. Communications and Control Engineering. Springer.

Zhu, L. M., Modares, H., Peen, G. O., Lewis, F. L., Yue, B., 2015. Adaptive suboptimal output-feedback control for linear systems using integral reinforcement learning. IEEE Transactions on Control Systems Technology 23 (1), 264-273. 